\title{
Distributing the Electron Dose to Minimise Electron Beam Damage in Scanning Transmission Electron Microscopy
}

Daniel Nicholls ${ }^{1}$, Juhan Lee ${ }^{1}$, Houari Amari ${ }^{1}$, Andrew Stevens ${ }^{2}$, B. Layla Mehdi ${ }^{1}$ and Nigel Browning ${ }^{2}$

${ }^{1}$ University of Liverpool, Liverpool, England, United Kingdom, ${ }^{2}$ Sivananthan Laboratories, Bolingbrook, Illinois, United States

Beam induced sample damage occurs during STEM $^{1}$ imaging when the electron dose and/or dose rate surpasses a sample dependant critical threshold ${ }^{2}$. Typically imaging resolution scales with cumulative electron dose and to capture high quality images higher electron doses are required. For STEM, the beam size and current (i.e. dose and dose rate in the beam) are the same at all magnifications, with the difference between low and high magnification being the spatial distribution of the irradiated spots in the scan. What does change is the spacing between probe positions in the raster scan, which decreases with increasing magnification, eventually getting to the situation where the electron beam and/or damage species diffusion profiles overlap (Figure 1) - this is the case for most high-resolution STEM image acquisitions. Simulations that compare raster, random, and line hop scanning during image acquisition in a homogenous film have been performed using the concept of beam influence - the electron beam imparts energy onto the sample, and it is the accumulation of this imparted energy which causes beam induced phenomena; such as radiolysis ${ }^{3}$, knock-on ${ }^{4}$, etc. By modelling the beam influence, these simulations can explain how increasing the magnification (reducing the step separation) causes a local increase in the maximum beam influence under the beam during raster scanning. The simulations also show how the alternative sampling schemes, random and line hop scanning, can be used to reduce the local maximum beam influence (Figure 2) with the goal being to find an imaging condition for a particular sample which produces the lowest maximum beam influence for the highest possible electron dose. These alternative sampling methods have traditionally not been used in STEM imaging for the simple reason that there is not enough information to achieve atomic resolution in a direct image. However, when we combine these alternative scanning methods with inpainting ${ }^{5}$, subsampling can be used to obtain fast (it uses fewer pixels to form the image), high quality, low dose and reduced damage high resolution STEM images. The use of inpainting, compressive sensing and machine learning for all forms of STEM imaging (HAADF, ABF, EDS, EELS and ptychography) will be described, and the potential limits of temporal and spatial resolution for STEM imaging will be discussed in this presentation. 

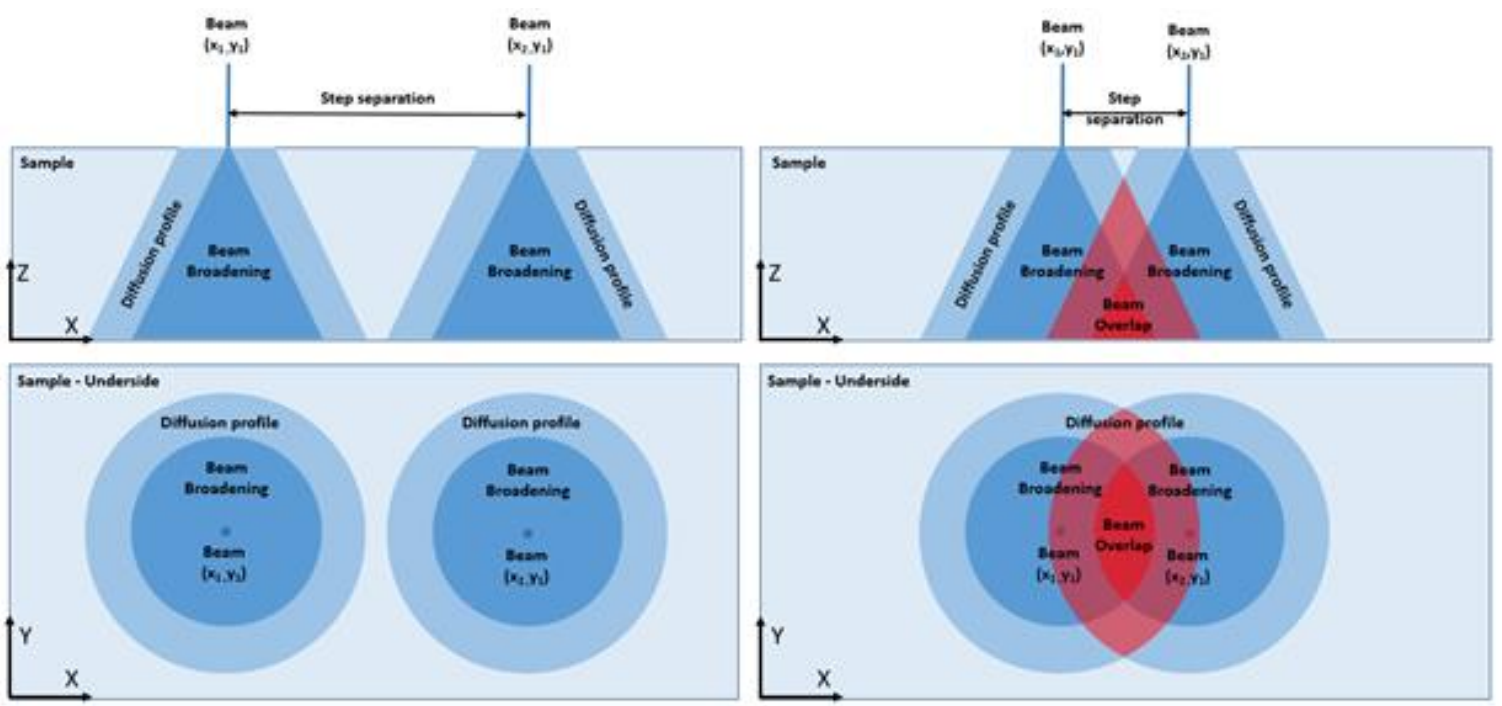

Figure 1. Small step separations between successive sampling points causes beam overlap due to beam broadening and/or diffusion effects (right). Increasing the step separation beyond a certain value reduces the effects of beam overlap (left).

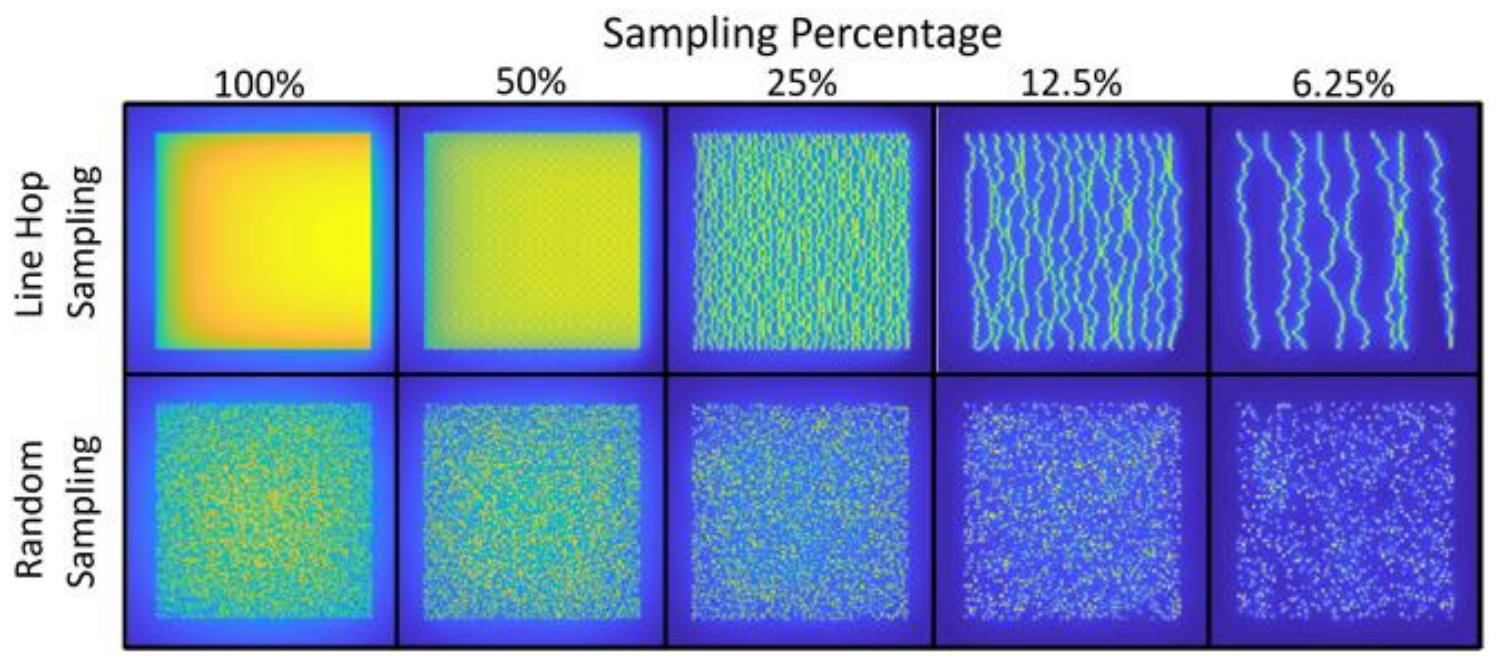

Figure 2. Line hop sampling provides an approximately equivalent distribution to random sampling at the same sampling percentage. The irradiated area is $128 \times 128$ pixels.

\section{References}

$1 \quad$ P. Batson, N. Dellby, and O. L. Krivanek, Nature 418, 617 (2002). ${ }^{2} \quad$ R. F. Egerton, P. Li, and M. Malac, Micron 35 (6), 399 (2004). ${ }^{3} \quad$ R. F. Egerton, Ultramicroscopy 127, 100 (2013). 4 H. Gu, G. Li, C. Liu, F. Yuan, F. Han, L. Zhang, and S. Wu, Sci Rep 7 (1), 184 (2017). 5 A. Stevens, H. Yang, L. Carin, I. Arslan, and N. D. Browning, Microscopy (Oxf) 63 (1), 41 (2014). 